\title{
La prosa de José Montero Iglesias en Prensa GRÁFICA
}

\author{
María Álvarez Villar \\ UNED
}

Title: The prose of José Montero Iglesias in Prensa Gráfica.

\begin{abstract}
This article lists all the prose publications that the writer José Montero Iglesias (Ciudad Rodrigo, I 878 - Navacerrada [Madrid] 1920) published for Prensa Gráfica between I9I I and I920, specifically those that were published in the magazines Mundo Grafico, Nuevo Mundo, La Esfera and Por esos mundos, with their respective links.
\end{abstract}

Key words: Montero Iglesias. Catalogue. Prose. Journalism. Literature. Prensa Gráfica.

A mi maestro, José Montero Reguera.

Según el Diccionario Biográfico Español (2018, web), José Montero Iglesias (1 878-1 920), fue un escritor cuya vocación literaria le llevó a escribir desde muy joven en gran número de revistas y periódicos cántabros como La Atalaya, Letras Montañesas o El Cantábrico. Años después, con su traslado a Madrid, comienza a formar parte de la editorial Prensa Gráfica donde publica periódicamente en cuatro de sus revistas: La Esfera, Nuevo Mundo, Mundo Gráfico y Por esos mundos. Su amigo y compañero José Francés lo describía del siguiente modo en Ahora (I I-02-1933, web):

Conviví con Montero Iglesias mucho tiempo en las redacciones de La Esfera y Mundo Gráfico. Sé bien cuánto hizo y cuánto la suerte no le consintió hacer. Entre las alternativas de una labor periodística obstinada y entusiasta fue realizando su labor literaria: versos, ensayos críticos, biografías de montañeses paisanos suyos, cuentos, comedias. La gloria, más cicatera que piadosa, tendía sobre aquellos esfuerzos vibrantes de un verdadero artista enfermo de cuerpo, pero recio de espíritu, fulgores tibios. Y él, hurañamente altivo, ni siquiera parecía notar el claror suave que venía a buscar el fondo oscuro de su existencia atormentada. (...) Montero Iglesias incorporó a la moderna pléyade de los portalises del primer decenio del XX una sinceridad expansiva, No disfrazó jamás el sentimiento ni resecó la sensibilidad con el conceptismo detallista. 
Montero Iglesias fue un literato muy apreciado por sus compañeros de profesión, quienes lo despiden después de su fallecimiento, añadiendo que era un "ilustre poeta y brillantísimo literato y periodista, redactor de Prensa Gráfica, con cuyos bellos y sentidos trabajos se han avalorado durante mucho tiempo las páginas de nuestras revistas" (Mundo Gráfico, 2 I-07-I 920 , web). De acuerdo con Dolores Sáiz (I 998: 200), estas publicaciones periódicas formaban parte de "una prensa consolidada, moderna, preocupada por todo tipo de temas y capaz de satisfacer las necesidades de información, formación y entretenimiento de sus lectores”, y, en ellas, Montero Iglesias publica gran cantidad de artículos y poemas a lo largo de su vida. Entre ellos, se pueden encontrar secciones como Palabras y palabras, Crónicas montañesas, Breviario de la guerra, Reporterismo pintoresco o Los caudillos, en las que el autor expresaba sus preocupaciones más profundas. Asimismo, sus publicaciones destacan por el gran conocimiento de los diferentes autores y personajes de interés como Zorrilla, Espronceda, Galdós o Rubén Dario, entre otros muchos. En ocasiones, haciendo honor a su gran amistad con Galdós, publica bajo el seudónimo galdosiano "Salvador Monsalud".

En este artículo se catalogan todas las publicaciones en prosa que Montero Iglesias redactó para la editorial Prensa Gráfica entre los años I9I I y I920, concretamente aquellas que se publicaron en las revistas Mundo Gráfico, Nuevo Mundo, La Esfera y Por esos mundos, con sus respectivos enlaces.

\section{A) Publicaciones en Mundo Gráfico:}

I 9 I 2

25/o9 - Galdós en "San Quintín” [p. 7: enlace]

27/I I - PALABRAS Y PALABRAS: La tierra lejana [p. 3: enlace]

I 8 / I 2 - PALABRAS Y PALABRAS: La noche santa [p. 3: enlace]

$25 / 12$ - PALABRAS Y PALABRAS: Balada del año viejo [p. 3: enlace]

I9I3

or/or - PALABRAS Y PALABRAS: Limosna de Reyes [p. 9: enlace]

22/or - PALABRAS Y PALABRAS: La ciudad lejana [p. 3: enlace] 
29/o - PALABRAS Y PALABRAS: El Príncipe Carnaval [p. 7: enlace]

o5/o2 - Miércoles de ceniza [p. 7: enlace]

I 2/o2 - PALABRAS Y PALABRAS: Campoamor [p. 9: enlace]

I 9/o2 - PALABRAS Y PALABRAS: Orden y método [p. 8: enlace]

26/o2 - PALABRAS Y PALABRAS: Páginas de un poeta [pp. 7-8: enlace]

o5/o3 - PALABRAS Y PALABRAS: Salve fémina [p. 7: enlace]

I 2/o3 - PALABRAS Y PALABRAS: Mi calle [p. 7: enlace]

i 9/o3 - PALABRAS Y PALABRAS: A modo de interviú [p. 9: enlace]

26/o3 - La señorita primavera [p. 7: enlace]

02/o4 - Evocación [p. 7: enlace]

o9/o4 - La vida quieta [p. 9: enlace]

I6/o4 - Por los niños [pp. 6-7: enlace]

30/o4 - Los velardes [p. s: enlace]

o7/os - El héroe [p. 6: enlace]

2 I/os - Páginas infantiles [pp. 6-7: enlace]

04/o6 - Deuda de gratitud [pp. 6-7: enlace]

I I/o6 - CRÓNICAS MONTAÑESAS: para empezar [p. 7: enlace]

25/o6 - Camino adelante [p. 7: enlace]

o2/o7 - CRÓNICAS MONTAÑESAS: El nuevo alcalde [p. 8: enlace]

o9/o7 - CRÓNICAS MONTAÑESAS: ¡Se respira! [pp. 8-9: enlace]

23/o7 - CRÓNICAS MONTAÑESAS: Un poeta del mar [Sobre José María y Escalante pp. 5-6: en o6/o8 - CRÓNICAS MONTAÑESAS: De la vida pintoresca; El valenciano

errante; La sombra de "Sotileza"; Desde el Turia al Cantábrico

pp. 8-9: enlace

ı/o9 - CRÓNICAS MONTAÑESAS: El Parnasillo de Basañez [p. 4: enlace]

i s/Io - CRÓNICAS MONTAÑESAS: Una joya artística [p. s: enlace]

I 2/ I I - Un nuevo cuadro de Goya [p. 6: enlace]

Io/ I 2 - La guardesa [p. 6: enlace]

3 I/ I 2 - La canción de los niños [p. 7: enlace]

I9I4

I4/or - Año nuevo: la tragedia de las hojas [p. 6: enlace]

25/o3 - GENTE QUE PASA: Mi amigo el provinciano [p. 9: enlace]

ı/o6 - El dosctor D. Ángel de Castanedo: Elogio de su vida

p. 4: enlace

24/o6 - Nuestro amigo el Tío Sam [p. 8: enlace] 
o /o7 - CARTA ABIERTA: Al Sr. D. César Francisco Ferrer, en Almería [p. 9: enlace] 22/o7 - Torneado [p. s: enlace]

os/o8 - POR TIERRAS MONTAÑESAS: LA ALEGRÍA ESTIVAL [p. s: enlace] o9/o9 - El dictador errante [p. 6: enlace]

I6/o9 - POR TIERRAS DE CANTABRIA: Los cariños del mar [p. 4: enlace]

23/o9 - Concha Espina: "La esfinge Maragata” [p. 8: enlace]

o7/ ı - NOTAS MARGINALES: Olor á chamusquina; La catedral de Reims;

Los terribles Vosgos [pp. 8-9: enlace]

I4/ Io - La bancarrota del pacifismo: Un rato de palique [p. 7: enlace]

04/I I - PALABRAS Y PALABRAS: Don Juan [p. 6: enlace]

I I/ I I - BREVIARIO DE LA GUERRA: el vino de España [p. 7: enlace]

o9/ I 2 - BREVIARIO DE LA GUERRA: la melancolía del Kaiser [p. 9: enlace]

30/ I 2 - BREVIARIO DE LA GUERRA: al acabarse el año [p. 7: enlace]

I9I 5

24/o2 - Lo que cuesta la guerra [pp. 8-1o: enlace]

07/o4 - Por supremacía comercial [p. 4-5: enlace]

i s/o9 - CRÓNICAS MONTAÑESAS: su majestad el boulevard [p. s: enlace]

29/o9 - REPORTERISMO PINTORESCO: agua fresca [pp. 4-5: enlace]

I 3 / I - REPORTERISMO PINTORESCO: el relojero alemán [pp. 4-s: enlace]

20/ Io - REPORTERISMO PINTORESCO: la «carroza di tutti» [p. 4: enlace]

I 7/ I I - REPORTERISMO PINTORESCO: bambalinas y cañones [p. s: enlace]

24/ I I - REPORTERISMO PINTORESCO: música militar [p. s: enlace]

o I/ I 2 - In memoriam: Félix Méndez [p. 7: enlace]

o8/ i 2 - LOS CAUDILLOS: Mackensen [pp. 4-5: enlace]

I 5/I 2 - LOS CAUDILLOS: el archiduque Federico [pp. 4-5: enlace]

22/ I 2 - Regalo de príncipes [p. I I: enlace]

29/ I 2 - LOS CAUDILLOS: Foch [pp. 4-5: enlace]

I 9 I 6

os/o r - LOS CAUDILLOS: Enver Bajá [p. 4: enlace]

I 2/or - LOS CAUDILLOS: Von Der Goltz [p. 7: enlace]

26/o - LIBROS MONTAÑESES: «roto casi el navío» [p. ıо: enlace]

ı6/o2 - LOS CAUDILLOS: Potioreck [pp. 4-5: enlace]

24/o5 - Los héroes de África [p. 3: enlace]

3 I/os - Flores de caridad [pp. 7-8: enlace]

I 9/o7 - El deán de Sigüenza [pp. 8-9: enlace] 
25/io - Los pueblos nuevos - Gijón [p. 3: enlace]

1917

03/o I - Los crímenes del veneno [pp. 9- I I: enlace]

B) Publicaciones en Nuevo Mundo:

I9I 5

29/Io - PIEDRAS Y BRONCES: El ingenioso hidalgo [p. s: enlace]

I 2/ I I - “La argentinita” y Raquel Meller [p. I 4: enlace]

Io/ I 2 - PIEDRAS Y BRONCES: Don Pedro Calderón [p. I s: enlace]

I7/ I 2 - Los olvidados de la sociedad [p. 25: enlace]

I9I6

I4/o I - Los príncipes en la guerra [p. s: enlace]

28/or - Un aniversario: la muerte de Zorrilla [p. 27: enlace]

I I/o2 - Ganarás el pan... Las lindas chicas del café [p. i 8: enlace]

I 8/o2 - La casa señorial [p. 4: enlace]

25/o2 - LOS CAUDILLOS: Castelnau [p. 3 i: enlace]

17/o3 - Tres emperadores [p. 27: enlace]

o7/o4 - LOS CAUDILLOS: El gran duque [p. Io : enlace]

2 I/o4 - El árbol seco [p. ıо: enlace]

os/os - La casa de velarde [p. 2 I: enlace]

02/o6 - Espronceda y Teresa [p. 8: enlace]

o9/o6 - Una fiesta española [p. 29: enlace]

i s/og - Horas del puerto: Cuando se pone el sol [p. 7: enlace]

03/ I I - La capa de Don Juan [p. 29: enlace]

29/ I 2 - Una fiesta de caridad: Los pobres de Madrid [p. I 3: enlace]

I 917

os/or - Versos de Rubén Dario: La viuda del poeta [p. 2 I: enlace]

27/o4 - La dulcísima Mirentxu [p. 20: enlace]

29/o6 - La "casa editorial Calleja" [pp. 6-7: enlace]

I $3 /$ o7 - El milagro de las rosas [p. 2: enlace]

ı/o8 - De una labor humanitaria: La piedad del rey [p. 9- I : enlace]

I7/o8 - La torre en ruinas [p. 24: enlace]

os/Io - La musa sotileza [p. 2: enlace]

I 918

25/or - Juan, el herrero [p. 3: enlace] 
I919

2 I/o2 - Curiosidades literarias: ¿Zorrilla, plagiario? [p. 28: enlace]

28/o3 - De la vida pueblerina: Una historia vulgar [pp. I I-I 2: enlace]

I I/o7 - Sierrapando, el burlón [p. 9: enlace]

I s/o8 - Las memorias de Estrañi: El poeta de las "pacotillas" [p. s: enlace]

1920

o9/o r - Lealtad de dos ingenios: Pérez Galdós y Estrañi [p. 23: enlace]

06/o2 - Los orejones de Liébana [p. 35: enlace]

C) Publicaciones en La Esfera:

I 9 I 4

24/o - Fantasía [p. i 9: enlace]

2 1/o2 - CUENTOS ESPAÑOLES: El gaitero de los beyos [pp. i 3-i 4: enlace]

I 8/o4 - CUENTOS ESPAÑOLES: La sombra de Verlaine [pp. i 9-20: enlace]

29/o8 - Margarita Xirgú y Pérez Galdós: Una tarde en "San Quintín”

p. iо: enlace

I7/ Io - DE LA TIERRA CANTÁBRICA: Las torres legendarias [pp. 24-25: enlace]

28/ I I - Un cuadro histórico: La rendición de Breda [pp. i6- I7: enlace]

I9I

o5/o6 - Los robles de la raza: El solitario de Provedaño [pp. I 3-I4: enlace]

I 3 / I - Los héroes anónimos [p. 29: enlace]

20/ I I - El caballero santiaguista [p. 3 r: enlace]

27/ I I - La villa arcaica [p. 26: enlace]

04/ I 2 - POR TIERRAS DE LEYENDA: La infantería española [p. 34: enlace]

I I/ I 2 - Las lanchas vuelven... [p. 25: enlace]

I $8 /$ I 2 - La última bruja [p. i 9: enlace]

I 916

o8/o I - Literatura infantil [p. I 9: enlace]

29/o - CUENTOS ESPAÑOLES: Vuelo de águilas [pp. i 4-i s: enlace]

I 2/o2 - Paisajes de España: Donde nace el Ebro [p. 33: enlace]

26/o2 - Mujeres de fantasía [p. 2 I: enlace]

I $5 / 04$ - El milagro del pan y los peces [p. i 8: enlace]

06/os - Cinematógrafo artístico [p. 8: enlace]

I7/o6 - Las nuevas tomadas [p. 20: enlace]

29/o7 - Los gigantes del autol [p. I 9: enlace]

o9/o9 - CUENTOS ESPAÑOLES: Carmenchu [pp. i6-i 7: enlace] 
30/o9 - ESPAÑA MONUMENTAL: La catedral de Sigüenza

- Salvador Monsalud [p. Iо- I I: enlace]

30/o9 - Camino adelante... [pp. 19-20: enlace]

I I/ I - El escenario de Marianela [p. 6: enlace]

I 8 / I - Bélgica, la buena: La piedad de una tradición [p. 8: enlace]

30/ I 2 - Campos de soledad [p. 28: enlace]

I9I7

o6/o - Las que sueñan y esperan - Salvador Monsalud [p. 26: enlace]

I3/or - ANTAÑO Y HOGAÑO: Los placeres de la caza [pp. i 2-I 3: enlace]

20/or - La moza que fue maya y princesa [p. 29: enlace]

27/or - RECUERDOS Y BELLEZAS DE ESPAÑA: El Real Monasterio de Valparaíso [p. 2o: enlace]

24/o2 - La coronación de Zorrilla [p. 20: enlace]

I 2/os - El lunes trágico y glorioso [p. 27: enlace]

o9/o6 - Don Julián "El Charro" [p. s: enlace]

I4/o7 - El tribunal de las aguas [p. 39: enlace]

21/07 - De viaje [p. 20: enlace]

28/o7 - La verdad es dolor: EL PECADO DE CURIOSIDAD [p. 26: enlace]

25/o8 - Uno, como hay muchos [p. 7: enlace]

oI/o9 - Regatas [p. 23: enlace]

06/ Io - Historia trágica [p. 23: enlace]

22/I2 - En el camino [p. 25: enlace]

I9I8

or/or - El madrigal de Sor Teresa (cuento) [pp. 47-48: enlace]

I2/or - Trafalgar [p. i6: enlace]

og/o2 - Los sueños de Petruca [p. i7: enlace]

o9/o3 - De la vida que pasa: Libertad económica [p. 4: enlace]

I 3/o7 - Nuevos días, nuevas costumbres [p. 25 : enlace]

${ }_{28} /$ I 2 - El año viejo [p. s: enlace]

I919

oI/o2 - Historia de un hidalgo [pp. 2 I-22: enlace]

o8/o2 - La musa de Gustavo Adolfo [p. I4: enlace]

05/o7 - Mujeres de Perda: corte de amor y dolor [p. Io: enlace]

I 2/o7 - Mientras llueve [p. I7: enlace]

16/o8 - Cuando ya se ha puesto el sol [p. 6: enlace]

29/ I I - Lo de todos los años [p. 24: enlace] 
1920

$25 /$ I 2 - El pastor del abuelo [enlace]

I 916

o I/o4 - DE LA ESPAÑA CABALLERESCA: Santillana, la arcaica - Salvador Monsalud [pp. 73-80: enlace]

oI/os - De la epopeya nacional [pp. 47-55: enlace]

\section{REFERENCIAS BIBLIOGRÁFICAS}

Dolores SÁIz, María, (1998) "La prensa madrileña en torno a I898”, Historia y Comunicación Social, n. 3, 195-200.

Francés, José, (1933) “Un montañés olvidado: el biógrafo de Pereda”, Ahora, año IV, n. 675, 9.

F. M., (1920) "Retratos de actualidad", Mundo Gráfico, año X, n. 455, i 8 .

Montero Padilla, José, (2018) “Biografía de José Montero Iglesias”, Diccionario Biográfico Espa-

Material on-line:

nol, web, accesible en: http://dbe.rah.es/biografias/67020/jose-montero-iglesias

Hemeroteca Digital. Biblioteca Nacional de España, accesible en: web

Recibido: julio de 2020 Aceptado: septiembre de 2020 